\section{Fatores associados ao aumento do consumo de cigarros durante a pandemia da COVID-19 na população brasileira}

\author{
Factors associated with increased cigarette \\ consumption in the Brazilian population \\ during the COVID-19 pandemic
}

\author{
Factores asociados al aumento del consumo \\ de tabaco durante la pandemia de COVID-19 \\ en la población brasileña
}

\author{
Deborah Carvalho Malta 1 \\ Crizian Saar Gomes 2 \\ Paulo Roberto Borges de Souza Júnior 3 \\ Celia Landmann Szwarcwald 3 \\ Marilisa Berti de Azevedo Barros 4 \\ Ísis Eloah Machado 5 \\ Dalia Elena Romero 3 \\ Margareth Guimarães Lima 4 \\ Alanna Gomes da Silva 1 \\ Elton Junio Sady Prates 1 \\ Laís Santos de Magalhães Cardoso 1 \\ Giseli Nogueira Damacena ${ }^{3}$ \\ André Oliveira Werneck 6 \\ Danilo Rodrigues Pereira da Silva 7 \\ Luiz Otávio Azevedo 3
}

doi: 10.1590/0102-311X00252220

\section{Resumo}

Medidas de distanciamento social adotadas em diversos países para mitigar o impacto da pandemia de COVID-19 podem acarretar efeitos indesejáveis sobre a saúde e o comportamento das populações. Este estudo objetivou investigar o comportamento de fumar na população adulta brasileira durante a pandemia de COVID-19 e analisar os fatores associados ao aumento do consumo de cigarro. Foi realizado um inquérito virtual e a amostra final correspondeu a 45.160 indivíduos. Foram utilizados pesos de pós-estratificação e calculadas as razões de prevalência $(R P)$ brutas e ajustadas por sexo, idade e escolaridade, e os respectivos intervalos de 95\% de confiança (IC95\%). Modelos de regressão de Poisson com variância robusta foram aplicados para a análise de associação entre o aumento do consumo de cigarros e as variáveis sociodemográficas e as relativas à adesão ao distanciamento social, qualidade do sono, estado de ânimo, alteração no trabalho e nos rendimentos. A prevalência de fumantes foi de 12\% (IC95\%: 11,1-12,9), dos quais 34\% relataram aumento no consumo de cigarros. Esse aumento foi maior entre as mulheres $(R P=1,27$; IC95\%: 1,01-1,59) e entre indivíduos com o Ensino Médio incompleto $(R P=1,35 ;$ IC95\%: 1,02-1,79). O aumento do consumo de cigarros esteve associado à piora da qualidade do sono, sentir-se isolado dos familiares, triste ou deprimido, ansioso, ficar sem rendimentos e pior avaliação do estado de saúde. Estratégias de promoção da saúde, de prevenção do uso e de incentivo à cessação do consumo de cigarros, bem como intervenções em saúde mental, devem ser continuadas e reforçadas no contexto de distanciamento social durante a pandemia de COVID-19.

\section{Correspondência}

D. C. Malta

Departamento de Enfermagem Materno-Infantil e Saúde Pública, Escola de Enfermagem, Universidade Federal de Minas Gerais.

Av. Alfredo Balena 190, 40 andar, sala 434, Belo Horizonte, $M G$ 30130-100, Brasil.

dcmalta@uol.com.br

1 Escola de Enfermagem, Universidade Federal de Minas Gerais, Belo Horizonte, Brasil.

2 Faculdade de Medicina, Universidade Federal de Minas

Gerais, Belo Horizonte, Brasil.

3 Instituto de Comunicação e Informação Científica e

Tecnológica em Saúde, Fundação Oswaldo Cruz, Rio de Janeiro, Brasil.

${ }_{4}^{4}$ Faculdade de Ciências Médicas, Universidade Estadual de Campinas, Campinas, Brasil.

5 Escola de Medicina, Universidade Federal de Ouro Preto, Ouro Preto, Brasil.

6 Faculdade de Saúde Pública, Universidade de São Paulo, São Paulo, Brasil.

7 Centro de Ciências Biológicas e da Saúde, Universidade Federal de Sergipe, São Cristóvão, Brasil. 


\section{Introdução}

A pandemia de COVID-19, causada pelo novo coronavírus (SARS-CoV-2) e reconhecida pela Organização Mundial da Saúde (OMS) em 11 de março de 2020 1, alterou diferentes aspectos da sociedade, interferindo no convívio social, na economia, no emprego, no aumento das desigualdades, nas rotinas e dinâmicas dos serviços de saúde 2. Para mitigar a propagação do vírus, salvar vidas e melhorar a resposta dos serviços de saúde, medidas de distanciamento social foram recomendadas pela OMS 1. Entretanto, tais determinações também podem resultar em efeitos negativos nas condições de vida e de saúde da sociedade 3,4, e em alterações comportamentais, como o início ou aumento do consumo de cigarros 5,6,7,8,9.

Em períodos de epidemias e distanciamento social, a separação dos entes queridos, dos amigos, a perda de liberdade, o medo de contrair a doença, as mudanças nas atividades de rotina e as perdas financeiras podem causar situações de angústia, ansiedade, depressão, estresse, insônia e irritabilidade 10,11,12, que por sua vez podem aumentar o desejo de fumar 7,13,14,15. Um estudo realizado na Austrália durante a pandemia de COVID-19 identificou associação entre o aumento do consumo de cigarros e comportamentos como depressão (odds ratio - OR ajustado $=1,09$; intervalo de $95 \%$ de confiança IC95\%: 1,04-1,13), ansiedade (OR ajustado = 1,12; IC95\%: 1,06-1,18) e estresse (OR ajustado = 1,10; IC95\%: 1,05-1,15) 7 .

Revisões sistemáticas apontam que, embora os resultados não sejam ajustados para outros fatores que podem afetar a progressão da COVID-19, é provável que o tabagismo esteja associado ao pior prognóstico da doença, maior necessidade de internação em unidade de terapia intensiva (UTI) e resultados adversos da COVID-19 16,17,18,19. Entre os fatores envolvidos na relação entre o fumo e a infecção por SARS-CoV-2 estão o aumento da enzima conversora de angiotensina tipo 2 (ECA2) na população de fumantes 20,21 , movimentos repetitivos "mão/boca" que aumentam a contaminação viral 16,22 e a piora no desempenho cardiorrespiratório 23. Destaca-se, ainda, que o tabagismo é um dos mais importantes fatores de risco para as doenças cardiovasculares e metabólicas 24,25 e comorbidades que, por sua vez, ampliam o risco de agravamento da COVID-19. Um estudo encontrou que fumantes com COVID-19 apresentam 3,25 vezes mais chances de desenvolver quadros mais graves da doença do que os não fumantes 26 .

Nesse sentido, considerando-se o efeito nocivo do hábito de fumar na saúde, iniciado ou agravado em processos epidêmicos e os riscos adicionais de tal comportamento na pandemia de COVID-19, torna-se necessário monitorar a ocorrência de tabagismo e de mudança do comportamento de fumar, a fim de fomentar e direcionar intervenções e políticas públicas de incentivo a comportamentos saudáveis durante a vigência das medidas de distanciamento social. Assim, objetivou-se investigar o comportamento de fumar na população adulta brasileira durante a pandemia da COVID-19, e analisar os fatores associados ao aumento do consumo de cigarros.

\section{Métodos}

Este estudo utilizou dados da pesquisa ConVid - Pesquisa de Comportamentos. É um inquérito virtual de saúde, realizado durante a pandemia de COVID-19, para avaliar as mudanças que ocorreram na vida dos adultos brasileiros relacionadas às medidas de distanciamento social adotadas. O presente estudo constitui uma parceria entre pesquisadores da Universidade Federal de Minas Gerais, Fundação Oswaldo Cruz e Universidade Estadual de Campinas. Os participantes preencheram o Termo de Consentimento Livre e Esclarecido (TCLE) e todas as respostas foram anônimas e sem qualquer tipo de identificação. A pesquisa foi aprovada pela Comissão Nacional de Ética em Pesquisa (parecer no 3.980.277).

A coleta de dados foi realizada entre 24 de abril e 24 de maio de 2020. Os participantes foram convidados a fazer parte da pesquisa por meio de um processo de amostragem em cadeia. Na primeira etapa, os 15 pesquisadores envolvidos na concepção ou desenvolvimento da investigação escolheram um total de 200 outros pesquisadores de diferentes estados do Brasil para participarem como respondentes. Além disso, cada um dos 15 pesquisadores escolheu outras 20 pessoas de sua rede social, perfazendo um total de 500 pessoas escolhidas. A essas pessoas, denominadas de "sementes", foi soli- 
citada a replicação do envio do link da pesquisa a pelo menos 12 pessoas de suas redes sociais, obedecendo a uma estratificação por sexo, faixa etária (18-39 anos; 40-59 anos; 60 anos ou mais) e nível de escolaridade (Ensino Médio incompleto ou menos; Ensino Médio completo ou mais). As pessoas convidadas pelas "sementes" compuseram a segunda onda da cadeia de recrutamento. A cada pessoa da segunda onda foi solicitado que convidasse pelo menos outras três de suas redes sociais, por meio da mensagem ao final do questionário: "Faça parte da Rede ConVid e compartilhe esta pesquisa com três ou mais convidados da sua rede social. Você pode fazer isso clicando aqui ou copiando e enviando o nosso link https://convid.fiocruz.br". As informações sobre o estudo foram divulgadas por meio de releases de imprensa, comunicações sociais das instituições de pesquisa participantes, departamentos estaduais de saúde e mídias sociais. Outrossim, o link da pesquisa também estava disponível nos sites das instituições às quais os pesquisadores responsáveis pela investigação são vinculados. Com esse processo, a rede de convidados aumentou rapidamente e a amostra final de respondentes foi de 45.160 indivíduos adultos (com 18 anos ou mais de idade) 27.

A pesquisa ConVid - Pesquisa de Comportamentos abordou temas como adesão às medidas de distanciamento social, perdas econômicas, doenças crônicas, alterações nos estilos de vida e no estado de ânimo, dentre outros. Mais informações sobre a metodologia da pesquisa podem ser encontradas em Szwarcwald et al. 27.

A variável desfecho avaliada neste estudo, "aumento do consumo de cigarros durante a pandemia", foi analisada por meio das seguintes questões: "O sr(a) é fumante?"; "Antes da pandemia, em média, quantos cigarros você costumava fumar por dia?”; e "Durante a pandemia, em média, quantos cigarros você passou a fumar por dia?”. As opções de respostas para as duas últimas questões eram: "Não fumava cigarros, só outros produtos"; "Menos que 1 por dia”; "De 1 a 9 cigarros"; "De 10 a 19 cigarros"; "De 20 a 29 cigarros"; "De 30 a 39 cigarros"; "40 ou mais cigarros". Para avaliar a quantidade de cigarros antes (tempo 1 ou t1) e durante a pandemia (tempo 2 ou t2) foi usado o ponto médio da categoria de resposta e calculada a diferença entre os dois momentos ( $\mathrm{t} 2 \mathrm{-t} 1)$. Considerou-se aumento no consumo de cigarros quando a diferença entre $\mathrm{t} 2 \mathrm{e} \mathrm{t} 1$ foi positiva.

Como variáveis explicativas compreenderam-se os seguintes fatores: adesão às medidas de distanciamento social; qualidade do sono; estado de ânimo (sentir-se isolado dos familiares, sentir-se triste ou deprimido, sentir-se ansioso ou nervoso); mudanças no trabalho ou emprego, nos rendimentos; e autoavaliação do estado de saúde, além das variáveis sociodemográficas (Quadro 1).

As seguintes variáveis sociodemográficas foram analisadas: sexo (masculino e feminino), faixa etária (18-39, 40-59 e 60 anos ou mais) e escolaridade (Ensino Fundamental completo ou menos; Ensino Médio completo; e Ensino Superior completo ou mais).

Tendo em vista que a amostra não foi probabilística, utilizou-se pesos de pós-estratificação com base nos dados da Pesquisa Nacional por Amostra de Domicílios de 2019, do Instituto Brasileiro de Geografia e Estatística (IBGE), a fim de obter, para a amostra, a mesma distribuição da população brasileira por Unidade da Federação, sexo, faixa etária, raça/cor e grau de escolaridade. Para avaliar os fatores associados ao aumento do consumo de cigarros foram calculadas razões de prevalência (RP) brutas e ajustadas por sexo, idade e escolaridade, e os respectivos IC95\%, utilizando modelos de regressão de Poisson com variância robusta.

Realizou-se o processamento das informações no software Stata versão 14 (https://www.stata. com), empregando o módulo survey, que considera os pesos de pós-estratificação.

\section{Resultados}

Entre os participantes do estudo, a maioria era do sexo feminino (53,6\%; IC95\%: 52,0-55,0), tinha entre 18 a 39 anos de idade (45,7\%; IC95\%: 44,3-47,1) e apresentava Ensino Médio completo (72,4\%; IC95\%: 71,3-73,5).

A prevalência de fumantes na população foi de 12\% (IC95\%: 11,1-12,9), sendo que 34\% dos fumantes relataram ter aumentado o consumo de cigarros (Figura 1). Do total de pessoas que aumentaram o consumo de cigarros, 6,4\% (IC95\%: 4,3-9,4) aumentaram até 5 cigarros por dia, 22,5\% (IC95\%: 19,625,7 ) cerca de 10 por dia e 5,1\% (IC95\%: 3,4-7,7) 20 cigarros ou mais por dia. 
Quadro 1

Descrição das variáveis explicativas.

\begin{tabular}{|c|c|c|}
\hline VARIÁVEIS & PERGUNTAS & CATEGORIAS \\
\hline $\begin{array}{l}\text { Adesão às medidas de distanciamento } \\
\text { social }\end{array}$ & $\begin{array}{l}\text { Durante a pandemia do novo coronavírus, } \\
\text { com que intensidade você fez (ou ainda está } \\
\text { fazendo) restrição do contato com as pessoas? }\end{array}$ & $\begin{array}{l}\text { 1) Nenhuma restrição: Não fiz nada, levei vida } \\
\qquad \text { normal } \\
\text { 2) Pouca restrição: Procurei tomar cuidados, } \\
\text { ficar à distância das pessoas, reduzir um pouco } \\
\text { o contato, não visitar idosos, mas continuei } \\
\text { trabalhando e saindo } \\
\text { 3) Restrição intensa: Fiquei em casa só saindo } \\
\text { para compras em supermercado e farmácia } \\
\text { 4) Restrição total: Fiquei rigorosamente } \\
\text { em casa, saindo só por necessidades de } \\
\text { atendimento à saúde }\end{array}$ \\
\hline Qualidade do sono & A pandemia afetou a qualidade do seu sono? & $\begin{array}{l}\text { 1) Não afetou, continuo dormindo bem } \\
\text { 2) Com a pandemia comecei a ter problemas } \\
\text { de sono } \\
\text { 3) Eu já tinha problemas de sono e eles } \\
\text { continuaram da mesma forma } \\
\text { 4) Eu já tinha problemas de sono e eles } \\
\text { pioraram bastante } \\
\text { 5) Os problemas de sono diminuíram com a } \\
\text { pandemia }\end{array}$ \\
\hline Sentir-se isolado dos familiares & $\begin{array}{l}\text { No período da pandemia, com que frequência } \\
\text { você se sentiu isolado(a) dos seus familiares ou } \\
\text { amigos próximos? }\end{array}$ & $\begin{array}{l}\text { 1) Nunca } \\
\text { 2) Poucas vezes } \\
\text { 3) Muitas vezes } \\
\text { 4) Sempre }\end{array}$ \\
\hline Sentir-se triste ou deprimido & $\begin{array}{c}\text { No período da pandemia, com que frequência } \\
\text { você se sentiu triste ou deprimido(a)? }\end{array}$ & $\begin{array}{l}\text { 1) Nunca } \\
\text { 2) Poucas vezes } \\
\text { 3) Muitas vezes } \\
\text { 4) Sempre }\end{array}$ \\
\hline Sentir-se ansioso ou nervoso & $\begin{array}{c}\text { No período da pandemia, com que frequência } \\
\text { você se sentiu ansioso(a) ou nervoso(a)? }\end{array}$ & $\begin{array}{l}\text { 1) Nunca } \\
\text { 2) Poucas vezes } \\
\text { 3) Muitas vezes } \\
\text { 4) Sempre }\end{array}$ \\
\hline Mudança no trabalho & $\begin{array}{l}\text { Como a pandemia afetou a sua ocupação/ } \\
\text { trabalho? }\end{array}$ & $\begin{array}{l}\text { 1) Manteve igual } \\
\text { 2) Começou a trabalhar } \\
\text { 3) Parou de trabalhar }\end{array}$ \\
\hline Mudança nos rendimentos familiares & Como a pandemia afetou a renda da família? & $\begin{array}{l}\text { 1) Manteve igual } \\
\text { 2) Aumentou } \\
\text { 3) Diminuiu } \\
\text { 4) Fiquei sem rendimentos }\end{array}$ \\
\hline Mudança no estado de saúde & $\begin{array}{l}\text { Você acha que a pandemia provocou } \\
\text { mudanças no seu estado de saúde? }\end{array}$ & $\begin{array}{l}\text { 1) Ficou igual } \\
\text { 2) Melhorou } \\
\text { 3) Piorou }\end{array}$ \\
\hline
\end{tabular}




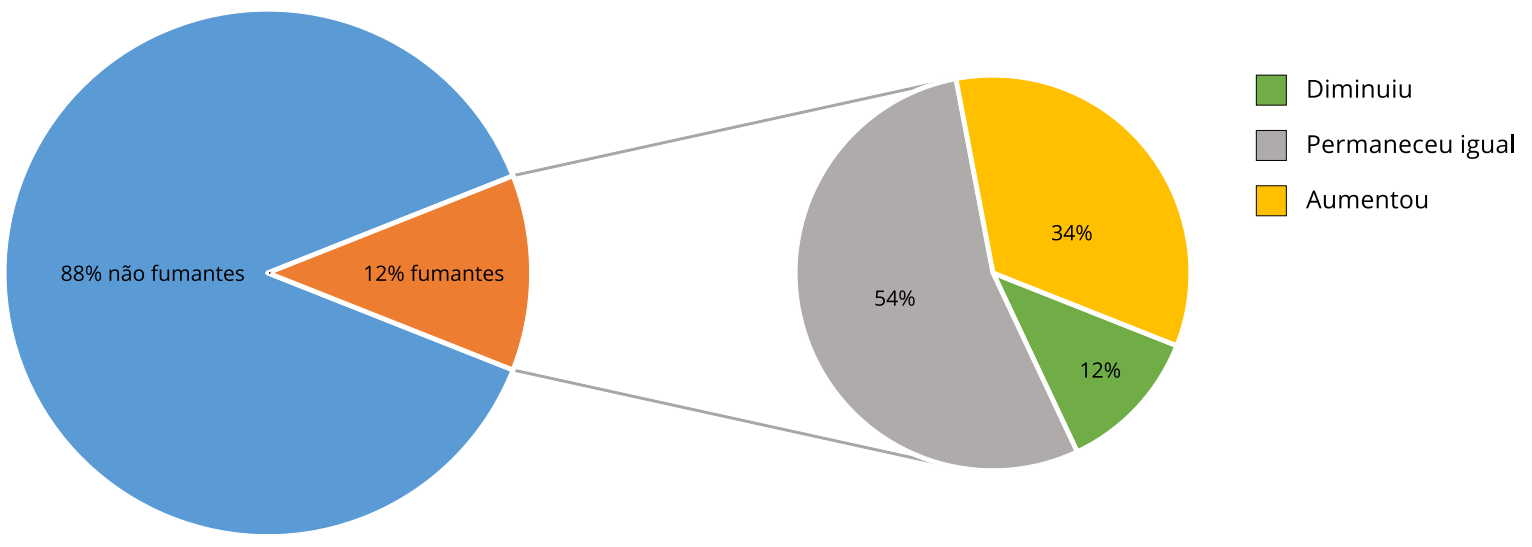

O aumento do consumo foi maior entre as mulheres ( $R P=1,3$; IC95\%: 1,1-1,6) e também entre aqueles com Ensino Médio completo ou menos ( $\mathrm{RP}$ = 1,3; IC95\%: 1,1-1,8), comparados aos que completaram o Ensino Superior ou mais. Não houve diferenças significativas com relação à faixa etária (Tabela 1).

A Tabela 2 apresenta os fatores associados ao aumento do consumo de cigarros durante a pandemia de COVID-19. No que diz respeito à qualidade do sono, verificou-se que, comparando com as pessoas que continuaram dormindo bem durante a pandemia, houve aumento no consumo de cigarros entre os indivíduos que começaram a ter problemas de sono durante a pandemia (RP ajustada $=$ 2,1; IC95\%: 1,4-3,0) ou que apresentaram piora nos problemas de sono (RP ajustada = 2,2; IC95\%: 1,5$3,2)$. Por outro lado, as pessoas que relataram que os problemas de sono diminuíram também reduziram o consumo de cigarros (RP ajsutada = 0,5; IC95\%: 0,2-0,9). No que se refere ao estado de ânimo, observou-se maior prevalência do aumento do consumo de cigarros entre as pessoas que relataram as seguintes situações: sentir-se isolado dos familiares por muitas vezes (RP ajustada $=2,00$; IC95\%: 1,2-3,4) ou sempre (RP ajustada = 2,4; IC95\%: 1,4-4,2); sentir-se triste ou deprimido por muitas vezes ( $\mathrm{RP}$ ajustada = 2,2; IC95\%: 1,4-3,7) ou sempre (RP ajustada = 2,4; IC95\%: 1,4-4,0); e sentir-se ansioso ou nervoso por muitas vezes (RP ajustada $=3,1$; IC95\%: 1,4-6,7) ou sempre (RP ajustada $=3,5$; IC95\%: 1,6-7,8). A redução da renda não alterou o consumo de cigarros, ao passo que ficar sem rendimentos aumentou o consumo (RP ajustada $=1,5 ;$ IC95\%: 1,1-2,0). O relato de piora do estado de saúde com base na autoavaliação de saúde, também esteve associado ao aumento do consumo de cigarros (RP ajustada $=1,5 ;$ IC95\%: 1,2-1,8). As variáveis adesão ao distanciamento social e mudança no trabalho/ emprego não estiveram associadas ao aumento do consumo de cigarros.

\section{Discussão}

Os achados do presente estudo apontaram uma prevalência de $12 \%$ de fumantes, dos quais cerca de um terço referiu ter aumentado o consumo de cigarros durante a pandemia. Em contrapartida, 12\% relataram ter reduzido o número de cigarros fumados e $54 \%$ permaneceram sem alteração. O aumento do uso de cigarros foi mais elevado entre as mulheres e também em indivíduos com Ensino Médio incompleto. Após ajuste por sexo, idade e escolaridade, os fatores associados ao aumento do consumo 
Tabela 1

Prevalência e razão de prevalência do aumento do consumo de cigarros durante a pandemia de COVID-19, segundo caraterísticas sociodemográficas. ConVid - Pesquisa de Comportamentos, Brasil, 2020.

\begin{tabular}{|c|c|c|c|c|}
\hline \multirow[t]{2}{*}{ Variáveis } & \multirow{2}{*}{$\begin{array}{c}\text { Total } \\
\%(\text { IC95\%) }\end{array}$} & \multicolumn{2}{|c|}{ Aumento do consumo de cigarro } & \multirow[t]{2}{*}{ RP (IC95\%) } \\
\hline & & $\begin{array}{c}\text { Não } \\
\%(\text { IC95\%) }\end{array}$ & $\begin{array}{c}\text { Sim } \\
\%(I C 95 \%)\end{array}$ & \\
\hline Total & & $66,0(62,1-69,6)$ & $34,0(30,4-37,9)$ & \\
\hline \multicolumn{5}{|l|}{ Sexo } \\
\hline Masculino & $46,4(45,0-47,9)$ & $69,8(63,8-75,2)$ & $30,2(24,8-36,1)$ & Referência \\
\hline Feminino & $53,6(52,0-55,0)$ & $61,6(56,7-66,3)$ & $38,4(33,7-43,3)$ & $1,3(1,1-1,6)$ \\
\hline \multicolumn{5}{|l|}{ Faixa etária (anos) } \\
\hline $18-39$ & $45,7(44,3-47,1)$ & $62,8(56,1-69,0)$ & $37,2(31,0-43,9)$ & $1,1(0,8-1,5)$ \\
\hline $40-59$ & $34,0(32,7-35,4)$ & $68,8(63,4-73,7)$ & $31,2(26,3-36,6)$ & $0,4(0,7-1,3)$ \\
\hline 60 anos ou mais & $20,3(19,1-21,6)$ & $67,0(58,1-74,9)$ & $33,0(25,1-41,9)$ & Referência \\
\hline \multicolumn{5}{|l|}{ Escolaridade } \\
\hline Ensino Fundamental completo ou menos & $11,1(10,1-12,3)$ & $55,6(43,3-67,3)$ & $44,4(32,7-56,7)$ & $1,3(1,1-1,8)$ \\
\hline Ensino Médio completo & $72,4(71,3-73,5)$ & $67,8(63,2-72,1)$ & $32,2(27,9-36,8)$ & $0,9(0,8-1,1)$ \\
\hline Ensino Superior completo ou mais & $16,5(16,0-17,0)$ & $67,1(64,8-69,4)$ & $32,9(30,6-35,2)$ & Referência \\
\hline
\end{tabular}

IC95\%: intervalo de 95\% de confiança; RP: razão de prevalência.

de cigarros foram: sentir-se isolado dos familiares, triste ou deprimido, ansioso, piora da qualidade do sono, ficar sem rendimentos e pior avaliação do estado de saúde.

Em um estudo conduzido na Austrália durante a pandemia de COVID-19, a prevalência de fumantes (11\%) foi semelhante à do presente estudo (12\%), entretanto, a maioria (89\%) dos entrevistados não mudou seu comportamento de fumar e a prevalência de aumento do consumo entre fumantes foi de apenas $6,9 \% 7$. Nesse mesmo estudo observaram-se outras mudanças negativas nos comportamentos, para além da piora no consumo do tabagismo, como a redução da prática de atividade física (48,9\%), a piora no sono (40,7\%) e o aumento do consumo de bebidas alcoólicas $(26,6 \%) 7$. Estudos realizados na Itália e nos Estados Unidos indicaram aumento da prevalência de tabagismo na população durante as recessões econômicas que ocorreram nestes países, atribuído ao suposto efeito de redução do estresse ocasionado pelos problemas financeiros e pelo desemprego 28,29 . No entanto, outro estudo realizado na Itália, entre 2016 e 2017, que investigou o efeito da crise econômica no estilo de vida, revelou redução do hábito de fumar em decorrência das restrições orçamentárias e do menor poder aquisitivo 30 . Nesse sentido, um período de crise pode, paradoxalmente, predispor ao aumento do tabagismo, dificultar a iniciação ou contribuir para a cessação do hábito 30 .

O estado de ânimo é um fator importante para o aumento do consumo do tabaco. Diversos estudos descrevem a relação entre uso de tabaco e afetividade negativa, angústia ou sensibilidade à ansiedade entre indivíduos em situações de estresse 7,31,32,33,34,35,36,37. Alexander et al. 32 descreveram que os sentimentos relacionados ao isolamento social estiveram associados ao uso do tabaco, ao identificar que os indivíduos investigados dobraram o seu consumo durante a crise causada pelo furacão Katrina, em Nova Orleans, nos Estados Unidos, que deixou milhares de pessoas isoladas. Ao analisar uma amostra de 279 fumantes, os mesmos autores identificaram que os negros aumentaram em duas vezes o consumo do tabaco e os estressores que mais influenciaram foram: sofrimento mental, menor apoio social e maior vulnerabilidade social e econômica. Um estudo de revisão sistemática, que examinou a comorbidade do transtorno devido ao estresse pós-traumático e uso de tabaco, revelou que a prevalência do uso de produtos de tabaco em indivíduos com transtorno devido ao estresse pós-traumático foi de 24\%, com altos níveis de dependência de nicotina e uso abusivo de produtos de tabaco 31 . O aumento do consumo abusivo entre os indivíduos com essa condição enfatiza a importância de estados emocionais negativos, como ansiedade, depressão e estresse, como fatores contribuintes para 
Tabela 2

Fatores associados ao aumento do consumo de cigarros durante a pandemia de COVID-19. ConVid - Pesquisa de Comportamentos, Brasil, 2020.

\begin{tabular}{|c|c|c|c|c|}
\hline \multirow[t]{3}{*}{ Variáveis } & \multicolumn{2}{|c|}{ Aumento do consumo de cigarro } & \multirow[t]{3}{*}{ RP bruta (IC95\%) } & \multirow[t]{3}{*}{ RP ajustada (IC95\%) * } \\
\hline & Não & Sim & & \\
\hline & $\%(I C 95 \%)$ & $\%(I C 95 \%)$ & & \\
\hline \multicolumn{5}{|l|}{ Intensidade da restrição social } \\
\hline Nenhuma restrição & $77,9(39,3-95,0)$ & $22,1(5,0-60,7)$ & Referência & Referência \\
\hline Pouca restrição & $65,7(57,2-73,3)$ & $34,3(26,7-42,8)$ & $1,5(0,4-5,9)$ & $1,4(0,4-5,4)$ \\
\hline Restrição intensa & $64,8(59,9-69,5)$ & $35,2(30,5-40,1)$ & $1,6(0,4-6,0)$ & $1,4(0,4-5,4)$ \\
\hline Restrição total & $69,9(60,5-77,9)$ & $30,1(22,1-39,5)$ & $1,4(0,3-5,2)$ & $1,2(0,3-4,8)$ \\
\hline \multicolumn{5}{|l|}{ Qualidade do sono } \\
\hline Não afetou, continuo dormindo bem & $79,4(72,1-85,2)$ & $20,6(14,8-27,9)$ & Referência & Referência \\
\hline Comecei a ter problemas de sono & $56,2(48,5-63,5)$ & $43,8(36,5-51,5)$ & $2,1(1,5-3,1)$ & $2,1(1,4-3,0)$ \\
\hline Continuei tendo os mesmos problemas de sono & $74,6(67,0-80,9)$ & $25,4(19,1-33,0)$ & $1,2(0,8-1,9)$ & $1,2(0,8-1,8)$ \\
\hline Problemas de sono pioraram bastante & $52,7(44,7-60,5)$ & $47,3(39,4-55,3)$ & $2,3(1,6-3,3)$ & $2,2(1,5-3,2)$ \\
\hline Problemas de sono diminuíram & $90,0(81,4-94,9)$ & $10,0(5,1-18,6)$ & $0,5(0,2-1,0)$ & $0,5(0,2-0,9)$ \\
\hline \multicolumn{5}{|l|}{ Sentir-se isolado dos familiares } \\
\hline Nunca & $83,1(72,5-90,2)$ & $16,9(9,8-27,5)$ & Referência & Referência \\
\hline Poucas vezes & $71,6(63,5-78,5)$ & $28,4(21,5-36,5)$ & $1,7(0,9-3,0)$ & $1,6(0,2-0,9)$ \\
\hline Muitas vezes & $63,3(57,4-68,8)$ & $36,7(31,2-42,6)$ & $2,2(1,3-3,7)$ & $2,0(1,2-3,4)$ \\
\hline Sempre & $54,5(45,8-62,8)$ & $45,5(37,2-54,2)$ & $2,8(1,5-4,7)$ & $2,4(1,4-4,2)$ \\
\hline \multicolumn{5}{|l|}{ Sentir-se triste ou deprimido } \\
\hline Nunca & $80,6(69,8-88,3)$ & $19,4(11,7-30,2)$ & Referência & Referência \\
\hline Poucas vezes & $76,4(70,8-81,2)$ & $23,6(18,8-29,1)$ & $1,2(0,7-2,0)$ & $1,2(0,7-2,0)$ \\
\hline Muitas vezes & $54,7(48,4-60,8)$ & $45,3(39,2-51,6)$ & $2,3(1,4-3,8)$ & $2,2(1,4-3,7)$ \\
\hline Sempre & $49,4(37,2-61,6)$ & $50,6(38,4-62,8)$ & $2,6(1,5-4,5)$ & $2,4(1,4-4,0)$ \\
\hline \multicolumn{5}{|l|}{ Sentir-se ansioso ou nervoso } \\
\hline Nunca & $86,9(72,9-94,2)$ & $13,1(5,8-27,1)$ & Referência & Referência \\
\hline Poucas vezes & $78,6(73,0-83,2)$ & $21,4(16,8-26,9)$ & $1,6(0,7-3,7)$ & $1,7(0,7-3,7)$ \\
\hline Muitas vezes & $58,9(52,9-64,6)$ & $41,1(35,4-47,1)$ & $3,1(1,4-6,9)$ & $3,1(1,4-6,7)$ \\
\hline Sempre & $50,3(40,5-60,0)$ & $49,7(40,0-59,5)$ & $3,8(1,4-6,9)$ & $3,5(1,6-7,8)$ \\
\hline \multicolumn{5}{|l|}{ Mudança no trabalho } \\
\hline Manteve igual & $68,2(63,7-72,5)$ & $31,8(36,3-37,5)$ & Referência & Referência \\
\hline Começou a trabalhar & $75,3(44,1-92,2)$ & $24,7(7,8-55,9)$ & $0,8(0,3-2,2)$ & $0,8(0,3-2,3)$ \\
\hline Parou de trabalhar & $60,3(61,8-69,4)$ & $39,7(32,6-47,2)$ & $1,2(0,9-1,6)$ & $1,2(0,9-1,5)$ \\
\hline \multicolumn{5}{|l|}{ Mudança na renda } \\
\hline Manteve igual & $70,7(63,9-76,7)$ & $29,3(23,3-36,0)$ & Referência & Referência \\
\hline Aumentou & $67,9(35,3-89,2)$ & $32,1(10,8-64,7)$ & $1,1(0,4-2,8)$ & $1,1(0,5-2,5)$ \\
\hline Diminuiu & $65,8(60,4-70,7)$ & $34,2(29,3-39,6)$ & $1,2(0,9-1,5)$ & $1,1(0,9-1,5)$ \\
\hline Ficou sem rendimentos & $53,8(42,6-64,5)$ & $46,2(35,5-57,4)$ & $1,6(1,1-2,2)$ & $1,5(1,1-2,0)$ \\
\hline \multicolumn{5}{|l|}{ Mudança estado de saúde } \\
\hline Manteve & $70,5(65,6-74,9)$ & $29,5(25,1-34,4)$ & Referência & Referência \\
\hline Melhorou & $75,5(52,1-89,8)$ & $24,5(25,1-34,4)$ & $0,8(0,4-1,8)$ & $0,9(0,4-1,9)$ \\
\hline Piorou & $54,5(48,1-60,9)$ & $45,5(30,1-51,9)$ & $1,5(1,2-1,9)$ & $1,5(1,2-1,8)$ \\
\hline
\end{tabular}

IC95\%: intervalo de 95\% de confiança; RP: razão de prevalência.

Nota: nenhuma restrição: não fez nada, levou vida norma; pouca restrição: procurou tomar cuidados, ficar à distância das pessoas, reduzir um pouco o contato, não visitar idosos, mas continuou saindo; restrição intensa: ficou em casa só saindo para compras em supermercado e farmácia; restrição total: ficou rigorosamente em casa, saindo só por necessidades de atendimento à saúde.

* RP ajustada por sexo, idade, escolaridade. 
o uso de tabaco 31. Pela perspectiva da neurofisiologia, há evidências da ação da nicotina sobre o sistema nervoso central, alterando o estado de ânimo ao desempenhar um papel na sensação de alívio do estresse, da angústia e da tristeza 38,39. Esse efeito de reforçador positivo seria mediado pelo sistema dopaminérgico mesolímbico 39 .

A piora do sono consiste em situação estressora e ansiogênica, o que também foi observado em um estudo realizado na província de Hubei, na China, com 939 indivíduos em isolamento social pela pandemia de COVID-19 40. De forma semelhante ao presente estudo, Altena et al. 41 identificaram a importância da piora do sono para o aumento do consumo de tabaco. O sono tem um importante papel na regulação emocional, e os distúrbios do sono acarretam mudanças no comportamento emocional 41 , o que pode resultar no aumento do consumo do tabaco.

Identificou-se também relação entre a perda dos rendimentos familiares e o aumento do uso de tabaco, possivelmente pelo fato de o evento ampliar a vulnerabilidade social das famílias e, consequentemente, a presença de estados emocionais negativos. A influência do trabalho e a perda de rendimentos sobre os distúrbios psicossociais têm sido igualmente bem documentadas na literatura internacional 42,43. Sabe-se que perder o emprego é considerada uma das experiências mais estressantes da vida adulta, levando à diminuição do poder de compra, à ruptura da família e à baixa autoestima 44, além de associar-se a mudanças no estado de saúde, bem como à depressão e ansiedade 45 . Destaca-se que a condição socioeconômica pregressa modula os efeitos negativos da pandemia, assim o impacto da COVID-19 será mais grave nas populações mais vulneráveis aos determinantes sociais adversos 46,47 .

Diante disso, torna-se importante avançar em estratégias de promoção da saúde direcionadas à adoção ou manutenção de comportamentos positivos relacionados à saúde, que devem ser utilizadas para lidar com o aumento do sofrimento psicológico durante a pandemia. É necessária uma abordagem abrangente, multissetorial e governamental para o controle do tabaco, que leve em consideração todos os pressupostos e compromissos da Convenção-Quadro para o Controle do Tabaco da OMS (CQCT-OMS) para deter a epidemia do tabaco e salvar vidas 48. A cessação do tabagismo por qualquer meio deve ser uma prioridade 6 .

Essa cessação pode ser difícil, especialmente em uma conjuntura adversa como na pandemia de COVID-19, tendo em vista que o acesso aos serviços de saúde pode ser comprometido. Adicionalmente, durante o distanciamento social imposto pela pandemia, o suporte presencial ofertado pelos profissionais de saúde pública aos tabagistas ficou prejudicado, havendo dificuldades para o início e a continuidade do tratamento nas unidades de saúde do Sistema Único de Saúde (SUS) brasileiro 49. Contribuem para isso tanto a orientação de sair de casa apenas em situações de extrema necessidade quanto a interrupção da oferta de ações presenciais - individuais e em grupo - na atenção primária à saúde, seja para que se evitem aglomerações ou mesmo em decorrência da sobrecarga dos serviços.

Nesse cenário, o fortalecimento de ações de educação em saúde por meio da Internet e do aconselhamento por telefone 50 tornam-se estratégias oportunas e complementares às medidas regulatórias estabelecidas global e nacionalmente. Uma diretriz fundamentada em revisões sistemáticas e meta-análises concluiu que o tratamento medicamentoso combinado ao aconselhamento com profissionais capacitados, seja individual, em grupo ou por telefone, são métodos altamente eficazes de cessação do tabagismo 51. Complementarmente, indicou que a eficácia do aconselhamento aumenta com o aumento da duração e do número de seções realizadas. São recomendadas também as intervenções por meio de materiais de autoajuda - panfletos, livretos, manuais, mensagens de e-mail, vídeos, áudios, programas de computador e website -, que, embora sejam menos eficazes que as opções anteriores, têm alto potencial de alcance do público-alvo e menor custo 51.

Na perspectiva das medidas regulatórias, ressalta-se a iniciativa implantada pela África do Sul, que proibiu a venda de produtos de tabaco e nicotina durante a pandemia de COVID-19, designando-os como bens não essenciais 52 . No âmbito das intervenções de educação em saúde, tanto a OMS quanto o Ministério da Saúde brasileiro, via Instituto Nacional de Câncer José Alencar Gomes da Silva (INCA), têm utilizado recursos virtuais com o objetivo de alertar os fumantes e pessoas expostas ao fumo passivo para os riscos associados à COVID-19, e encorajar a cessação do hábito durante a pandemia.

A OMS lançou, recentemente, um projeto de controle do tabagismo 53. Essa nova Iniciativa de Acesso para Cessação do Tabaco (IACT) visa à provisão de acesso gratuito e suporte à adesão à tera- 
pia de reposição da nicotina, e acesso à Florence, uma assistente virtual que auxiliará o usuário da interface no desenvolvimento individual de plano de cessação e responderá a dúvidas relacionadas ao tema tabagismo e COVID-19 53. Cumpre destacar a campanha do Dia Mundial sem Tabaco, promovida anualmente pela OMS no mês de maio que, em 2020, teve como objetivo conscientizar os jovens sobre as táticas de manipulação da indústria tabageira 54 .

No Brasil, a campanha mundial foi trabalhada com a temática Tabagismo e Coronavírus (COVID-19), dado o papel do tabagismo como potencial fator de risco para a COVID-19 e para o agravamento dos casos. Nacionalmente, ressalta-se ainda a campanha do Dia Nacional de Combate ao Fumo, realizada desde 1986 no mês de agosto, que, em 2020, manteve alinhamento temático com a campanha mundial e constituiu uma segunda fase da ação intitulada Coronavírus: Mais um Motivo Para Você Parar de Fumar 55. Foram elaboradas peças gráficas e textos de apoio para a veiculação em website e mídias sociais, reforçando a importância do não fumar e da adoção de hábitos saudáveis, bem como incentivando à cessação e desestimulando a iniciação do consumo no contexto da pandemia. Além disso, diante das dificuldades para iniciar e dar continuidade ao tratamento de fumantes nas unidades de saúde do SUS, decorrentes das medidas de distanciamento social, o INCA preparou uma websérie de oito vídeos curtos, cujo conteúdo abordou os prejuízos causados pelo tabagismo ${ }^{49}$. A instituição também publicou um alerta à população sobre os riscos do tabagismo para o agravamento da crise sanitária, no qual disponibilizou uma lista de orientações sobre o que pode ser feito individualmente para cessar o tabagismo 56. Uma nota de esclarecimento à Rede Nacional de Coordenadores do Programa Nacional de Controle do Tabagismo publicada no site da instituição reforçou a importância de garantir que os tabagistas que já estão em tratamento individual ou em grupo estejam com a medicação para o período, e de dar continuidade às orientações terapêuticas em grupo ou individual por meio de mensagens telefônicas ou outra ferramenta de comunicação 57.

Este é o primeiro estudo a investigar o comportamento de fumar na população adulta brasileira durante a pandemia da COVID-19 e a analisar os fatores associados ao aumento do consumo de cigarros. A inclusão de vários comportamentos de saúde, um grande tamanho amostral e a oportunidade na coleta de dados de forma virtual, considerando-se o cenário de pandemia, a rapidez nas respostas e baixo custo, são pontos fortes do presente estudo. No entanto, também existem algumas limitações a serem consideradas. Em primeiro lugar, todos os dados são autorrelatados, o que significa que as respostas estão sujeitas a viés de memória. Em segundo, os dados são transversais e, portanto, a causalidade não pode ser inferida. Em terceiro lugar, devido ao desenho amostral não probabilístico, os participantes do presente estudo possuem maior escolaridade e acesso à Internet, o que necessitou a utilização de pesos de pós-estratificação para a análise da base de dados. Consequentemente, a generalização para outras populações precisa ser confirmada por outros estudos. Além disso, recomendase a realização de estudos longitudinais para avaliar o impacto das restrições sociais nas mudanças no comportamento de fumar ao longo do tempo.

\section{Considerações finais}

Os dados apresentados sugerem que a piora da saúde mental, da qualidade do sono, da autoavaliação do estado de saúde e a ausência de rendimentos são fatores associados ao aumento do consumo de cigarros em adultos brasileiros durante a pandemia de COVID-19. Por conseguinte, ações de promoção da saúde direcionadas à adoção ou manutenção de comportamentos saudáveis, como mensagens direcionadas em mídias sociais e orientações pelos profissionais de saúde, especialmente da atenção primária à saúde, devem ser usadas para mitigar os sofrimentos psíquicos e os comportamentos não saudáveis, como o tabagismo. Além disso, estratégias para a prevenção do uso do tabaco e a promoção da cessação do fumo devem ser continuadas e reforçadas no período de vigência das medidas de distanciamento social, para que os ônus oriundos do consumo do tabaco não agravem ainda mais a situação de saúde da população no contexto de pandemia da COVID-19. 


\section{Colaboradores}

Todos os autores contribuíram na concepção e projeto do estudo, análise e interpretação dos dados, redação e revisão crítica do artigo, e aprovaram a versão final a ser publicada, sendo responsáveis por todos os aspectos do trabalho na garantia da exatidão e integridade de qualquer parte da obra.

\section{Informações adicionais}

ORCID: Deborah Carvalho Malta (0000-00028214-5734); Crizian Saar Gomes (0000-0001-65864561); Paulo Roberto Borges de Souza Júnior (00000002-8142-4790); Celia Landmann Szwarcwald (0000-0002-7798-2095); Marilisa Berti de Azevedo Barros (0000-0003-3974-195X); Ísis Eloah Machado (0000-0002-4678-2074); Dalia Elena Romero (0000-0002-2643-9797); Margareth Guimarães Lima (0000-0001-6996-0745); Alanna Gomes da Silva (0000-0003-2587-5658); Elton Junio Sady Prates (0000-0002-5049-186X); Laís Santos de Magalhães Cardoso (0000-0002-1114-5470); Giseli Nogueira Damacena (0000-0002-7059-3353); André Oliveira Werneck (0000-0002-9166-4376); Danilo Rodrigues Pereira da Silva (0000-00033995-4795); Luiz Otávio Azevedo (0000-00024876-5948).

\section{Referências}

1. World Health Organization. WHO DirectorGeneral's opening remarks at the media briefing on COVID-19 - 11 March 2020. https:// www.who.int/dg/speeches/detail/who-direc tor-general-s-opening-remarks-at-the-mediabriefing-on-covid-19---11-march-2020 (acessado em 20/Ago/2020).

2. Cucinotta D, Vanelli M. WHO declares COVID-19 a pandemic. Acta Biomed 2020; 91:157-60.

3. Barreto ML, Barros AJD, Carvalho MS, Codeço CT, Hallal PRC, Medronho RA, et al. O que é urgente e necessário para subsidiar as políticas de enfrentamento da pandemia de COVID-19 no Brasil? Rev Bras Epidemiol 2020; 23:e200032.

4. Holmes EA, O'Connor RC, Perry VH, Tracey I, Wessely S, Arseneault L, et al. Multidisciplinary research priorities for the COVID-19 pandemic: a call for action for mental health science. Lancet Psychiatry 2020; 7:547-60.

5. Alla F, Berlin I, Nguyen-Thanh V, Guignard R, Pasquereau A, Quelet S, et al. Tobacco and COVID-19: a crisis within a crisis? Can J Public Health 2020; 111:995-9.

6. Malta DC, Szwarcwald CL, Gomes CS, Machado IE, Souza Júnior PRB, Romero DE, et al. The COVID-19 Pandemic and changes in adult Brazilian lifestyles: a cross-sectional study, 2020. Epidemiol Serv Saúde 2020; 29:e2020407.

7. Stanton R, To QG, Khalesi S, Williams SL, Alley SJ, Thwaite TL, et al. Depression, anxiety and stress during COVID-19: associations with changes in physical activity, sleep, tobacco and alcohol use in Australian adults. Int J Environ Res Public Health 2020; 17:4065.

8. Chen DT. The psychosocial impact of the COVID-19 pandemic on changes in smoking behavior: evidence from a nationwide survey in the UK. Tob Prev Cessat 2020; 6:59.

9. Yach D. Tobacco use patterns in five countries during the COVID-19 lockdown. Nicotine Tob Res 2020; 22:1671-2.

10. Brooks SK, Webster RK, Smith LE, Woodland L, Wessely S, Greenberg N, et al. The psychological impact of quarantine and how to reduce it: rapid review of the evidence. Lancet 2020; 395:912-20.

11. Ornell F, Schuch JB, Sordi AO, Kessler FHP. "Pandemic fear" and COVID-19: mental health burden and strategies. Braz J Psychiatry 2020; 42:232-5.

12. Shigemura J, Ursano RJ, Morganstein JC, Kurosawa M, Benedek DM. Public responses to the novel 2019 coronavirus $(2019-\mathrm{nCoV})$ in Japan: mental health consequences and target populations. Psychiatry Clin Neurosc 2020; 74:281-2.

13. van Zyl-Smit RN, Richards G, Leone FT. Tobacco smoking and COVID-19 infection. Lancet Respir Med 2020; 8:664-5. 
14. McKay D, Yang H, Elhai J, Asmundson GJG. Anxiety regarding contracting COVID-19 related to interoceptive anxiety sensations: the moderating role of disgust propensity and sensitivity. J Anxiety Disord 2020; 73:102233.

15. Pericot-Valverde I, Elliott RJ, Miller ME, Tidey JW, Gaalema DE. Posttraumatic stress disorder and tobacco use: a systematic review and meta-analysis. Addict Behav 2018; 84:238-47.

16. Berlin I, Thomas D, Anne-Laurence LF, Cornuz J. COVID-19 and smoking. Nicotine Tob Res 2020; 22:1650-2.

17. Vardavas CI, Nikitara K. COVID-19 and smoking: a systematic review of the evidence. Tob Induc Dis 2020; 18:20.

18. Zhao Q, Meng M, Kumar R, Wu Y, Huang J, Lian $\mathrm{N}$, et al. The impact of COPD and smoking history on the severity of Covid-19: a systemic review and meta-analysis. J Med Virol 2020; 92:1915-21.

19. Banerjee B, Banerjee R. Role of tobacco in SARS-CoV-2 infection and COVID-19: a scoping review. Int J Non-Commun Dis 2020; 5:70-5.

20. Olds JL, Kabbani N. Is nicotine exposure linked to cardiopulmonary vulnerability to COVID-19 in the general population? FEBS J 2020; 287:3651-5.

21. Leung JM, Yang CX, Tam A, Shaipanich T, Hackett TL, Singhera GK, et al. ACE-2 expression in the small airway epithelia of smokers and COPD patients: implications for COVID-19. Eur Respir J 2020; 55:2000688.

22. Silva ALO, Moreira JC, Martins SR. COVID-19 and smoking: a high-risk association. Cad Saúde Pública 2020; 36:e00072020.

23. Cattaruzza MS, Zagà V, Gallus S, D’Argenio P, Gorini G. Tobacco smoking and COVID-19 pandemic: old and new issues. A summary of the evidence from the scientific literature. Acta Biomed 2020; 91:106-12.

24. World Health Organization. Global action plan for the prevention and control of NCDs 2013-2020. Geneva: World Health Organization; 2013

25. Malta DC, Silva AG, Machado IE, Sá ACMGN, Santos FM, Prates EJS, et al. Trends in smoking prevalence in all Brazilian capitals between 2006 and 2017. J Bras Pneumol 2019; 45:e20180384.

26. Guan W, Ni Z, Hu Y, Liang W, Ou C, He J, et al. Clinical characteristics of coronavirus disease 2019 in China. N Engl J Med 2020; 382:170820.

27. Szwarcwald CL, Souza Júnior PRB, Damacena GN, Malta DC, Barros MBA, Romero DE, et al. ConVid - Pesquisa de Comportamentos pela Internet durante a pandemia de COVID-19 no Brasil: concepção e metodologia de aplicação. Cad Saúde Pública 2021; 37:e00268320.

28. Mattei G, De Vogli R, Ferrari S, Pingani L, Rigatelli M, Galeazzi GM. Impact of the economic crisis on health-related behaviors in Italy. Int J Soc Psychiatry 2017; 63:649-56.
29. Gallus S, Ghislandi S, Muttarak R, Bosetti C. Effects of the economic crisis on smoking prevalence and number of smokers in the USA. Tob Control 2015; 24:82-8.

30. Petrelli F, Grappasonni I, Peroni A, Kracmarova L, Scuri S. Survey about the potential effects of economic downturn on alcohol consumption, smoking and quality of life in a sample of Central Italy population. Acta Biomed 2018; 89:93-8.

31. Pericot-Valverde I, Elliott RJ, Miller ME, Tidey JW, Gaalema DE. Posttraumatic stress disorder and tobacco use: a systematic review and meta-analysis. Addict Behav 2018; 84:238-47.

32. Alexander AC, Ali J, McDevitt-Murphy ME, Forde DR, Stockton M, Read M, et al. Racial differences in posttraumatic stress disorder vulnerability following hurricane Katrina among a sample of adult cigarette smokers from New Orleans. J Racial Ethn Health Disparities 2017; 4:94-103.

33. Asnaani A, Alpert E, McLean CP, Foa EB. Resilient but addicted: the impact of resilience on the relationship between smoking withdrawal and PTSD. J Psychiatr Res 2015; 65:146-53.

34. Asnaani A, Farris SG, Carpenter JK, Zandberg LJ, Foa EB. The relationship between anxiety sensitivity and posttraumatic stress disorder: what is the impact of nicotine withdrawal? Cogn Ther Res 2015; 39:697-708.

35. Baggett TP, Campbell EG, Chang Y, Magid LM, Rigotti NA. Posttraumatic stress symptoms and their association with smoking outcome expectancies among homeless smokers in Boston. Nicotine Tob Res 2016; 18:1526-32.

36. Fu SS, McFall M, Saxon AJ, Beckham JC, Carmody TP, Baker D, Joseph AM. Post-traumatic stress disorder and smoking: a systematic review. Nicotine Tob Res 2007; 9:1071-84.

37. Beckham JC, Calhoun PS, Dennis MF, Wilson SM, Dedert EA. Predictors of lapse in first week of smoking abstinence in PTSD and non-PTSD smokers. Nicotine Tob Res 2013; 15:1122-9.

38. Sociedade Brasileira de Pneumologia e Tisiologia. Tabagismo - o tabaco, exercícios aeróbicos, corridas e o coração. https://pesquisa. bvsalud.org/controlecancer/resource/pt/lisLISBR1.1-46463 (acessado em 20/Ago/2020).

39. Planeta CS, Cruz FC. Bases neurofisiológicas da dependência do tabaco. Rev Psiquiatr Clín 2005; 32:251-8.

40. Yuan S, Liao Z, Huang H, Jiang B, Zhang X, Wang Y, et al. Comparison of the indicators of psychological stress in the population of Hubei Province and non-endemic Provinces in China during two weeks during the coronavirus disease 2019 (COVID-19) outbreak in February 2020. Med Sci Monit 2020; 26:e9237671-e923767-10. 
41. Altena E, Baglioni C, Espie CA, Ellis J, Gavriloff D, Holzinger B, et al. Dealing with sleep problems during home confinement due to the COVID-19 outbreak: practical recommendations from a task force of the European CBT-I Academy. J Sleep Res 2020; 29:e13052.

42. Pikhart H, Bobak M, Siegrist J, Pajak A, Rywik S, Kyshegyi J, et al. Psychosocial work characteristics and self rated health in four postcommunist countries. J Epidemiol Community Health 2001; 55:624-30.

43. Stansfeld SA, Fuhrer R, Shipley MJ, Marmot MG. Work characteristics predict psychiatric disorder: prospective results from the Whitehall II Study. Occup Environ Med 1999; 56:302-7.

44. Ensminger ME, Celentano DD. Unemployment and psychiatric distress: social resources and coping. Soc Sci Med 1988; 27:239-47.

45. D’Souza RM, Strazdins L, Lim LL, Broom DH, Rodgers B. Work and health in a contemporary society: demands, control, and insecurity. J Epidemiol Community Health 2003; 57:84954.

46. Abrams EM, Szefler SJ. COVID-19 and the impact of social determinants of health. Lancet Respir Med 2020; 8:659-61.

47. Campos GWS. O pesadelo macabro da Covid-19 no Brasil: entre negacionismos e desvarios. Trab Educ Saúde 2020; 18:e00279111.

48. Al-Mandhari A, Hammerich A, El-Awa F, Bettcher D, Mandil A. Full implementation of the WHO Framework Convention on Tobacco Control in the Eastern Mediterranean Region is the responsibility of all. East Mediterr Health J 2020; 26:4-5.

49. Secretaria de Atenção Primária à Saúde, Ministério da Saúde. Websérie alerta população sobre os males do tabagismo. http://aps.sau de.gov.br/noticia/10258 (acessado em 25/ Nov/2020).

50. United Nations. Responding to the challenge of non-communicable diseases. Geneva: World Health Organization; 2019.

51. Clinical Practice Guideline Treating Tobacco Use and Dependence 2008 Update Panel, Liaisons, and Staff. A clinical practice guideline for treating tobacco use and dependence: 2008 update. A U.S. Public Health Service report. Am J Prev Med 2008; 35:158-76.
52. Egbe CO, Ngobese SP. COVID-19 lockdown and the tobacco product ban in South Africa. Tob Induc Dis 2020; 18:39.

53. World Health Organization. WHO and partners to help more than 1 billion people quit tobacco to reduce risk of COVID-19. https://www.who.int/news-room/detail/1007-2020-who-and-partners-to-help-morethan-1-billion-people-quit-tobacco-toreduce-risk-of-covid-19 (acessado em 20/ Ago/2020).

54. World Health Organization. World no tobacco day - 31 May 2020. Protecting youth from industry manipulation and preventing them from tobacco and nicotine use. https://www.who.int/news-room/events/de tail/2020/05/31/default-calendar/world-notobacco-day-2020-protecting-youth-fromindustry-manipulation-and-preventing-themfrom-tobacco-and-nicotine-use (acessado em 25/Nov/2020).

55. Instituto Nacional de Câncer José Alencar Gomes da Silva. Dia Nacional de Combate ao Fumo 2020: tabagismo e coronavírus (COVID-19) - segunda fase. https://www.in ca.gov.br/sites/ufu.sti.inca.local/files//media/ document//nota-tecnica-dia-nacional-decombate-ao-fumo-coronavirus.pdf (acessado em 25/Nov/2020).

56. Instituto Nacional de Câncer José Alencar Gomes da Silva. Alerta do INCA à população sobre tabagismo e coronavírus. https://www. inca.gov.br/publicacoes/notas-tecnicas/alertado-inca-populacao-sobre-tabagismo-e-coro navirus (acessado em 25/Nov/2020).

57. Instituto Nacional de Câncer José Alencar Gomes da Silva. Esclarecimento à Rede Nacional de Coordenadores do Programa Nacional de Controle do Tabagismo - Programa de Cessação do Tabagismo. https://www.inca.gov.br/ publicacoes/notas-tecnicas/esclarecimentorede-nacional-de-coordenadores-do-progra ma-nacional-de (acessado em 25/Nov/2020). 
Abstract

Social distancing measures adopted in various countries to mitigate the impact of the COVID-19 pandemic can lead to unwanted effects on their populations' health and behaviors. This study aimed to investigate smoking behavior in the Brazilian adult population during the COVID-19 pandemic and analyze factors associated with the increase in cigarette consumption. An online survey was performed, and the final sample included 45,160 individuals. The study used post-stratification weights and calculated crude prevalence ratios $(P R)$ and adjusted by sex, age, and schooling, and respective $95 \%$ confidence intervals (95\%CI). Poisson regression models with robust variance were applied to analyze associations between increased cigarette consumption and sociodemographic variables and adherence to social distancing, quality of sleep, state of mind, and changes in work and earnings. Prevalence of smokers was 12\% (95\% CI: 11.1-12.9), 34\% of whom reported an increase in cigarette consumption. The increase was greater among women (PR $=1.27$; 95\%CI: 1.01-1.59) and individuals with incomplete secondary schooling $(P R=1.35 ; 95 \% C I: 1.02-1.79)$. The increase in cigarette consumption was associated with worse quality of sleep, feeling isolated from family members or sad, depressed, or anxious, loss of earnings, and worse self-rated health. Health promotion strategies, smoking prevention, and encouragement for smoking cessation, as well as mental health interventions, should be continued and reinforced in the context of social distancing during the COVID-19 pandemic.

COVID-19; Quarentine; Health Risk Behaviors; Tobacco Use Disorders; Risk Factores

\section{Resumen}

Las medidas de distanciamiento social adoptadas en diversos países para mitigar el impacto de la pandemia de COVID-19 pueden acarrear efectos indeseables sobre la salud y el comportamiento de las poblaciones. Este estudio tuvo como objetivo investigar el comportamiento de fumar en la población adulta brasileña, durante la pandemia de COVID-19, y analizar los factores asociados al aumento del consumo de tabaco. Se realizó una encuesta virtual y la muestra final correspondió a 45.160 individuos. Se utilizaron pesos de posestratificación y se calcularon las razones de prevalencia (RP) brutas y ajustadas por sexo, edad y escolaridad, así como los respectivos intervalos de 95\% de confianza (IC95\%). Se aplicaron modelos de regresión de Poisson con variancia robusta para el análisis de asociación entre el aumento del consumo de tabaco y las variables sociodemográficas, así como las relativas a la adhesión al distanciamiento social, calidad del sueño, estado de ánimo, cambios en el trabajo e ingresos. La prevalencia de fumadores fue de un 12\% (IC95\%: 11,1-12,9), de los cuales un $34 \%$ relataron un aumento en el consumo de cigarrillos. Este aumento fue mayor entre las mujeres $(R P=1,27$; IC95\%: $1,01-1,59) y$ entre individuos con la enseñanza media incompleta $(R P=$ 1,35; IC95\%: 1,02-1,79). El aumento del consumo de tabaco estuvo asociado con un empeoramiento de la calidad del sueño, sentirse aislado de los familiares, triste o deprimido, ansioso, quedarse sin ingresos, al igual que con una peor evaluación del estado de salud. Las estrategias de promoción de salud, prevención del consumo y de alicientes para dejar el hábito de fumar, así como intervenciones en salud mental, deben ser continuas y estar reforzadas en el contexto de distanciamiento social durante la pandemia de COVID-19.

COVID-19; Cuarentena; Conductas de Riesgo para la Salud; Tabaquismo; Factores de Riesgo

Recebido em 25/Ago/2020

Versão final reapresentada em 26/Nov/2020

Aprovado em 01/Mar/2021 